\title{
Methods of Characterisation of DC Partial Discharge in Polymeric Cable Insulation
}

\author{
Euan A. Morris, W.H. Siew, Martin J. Given \\ Department of Electronic \& Electrical Engineering, University of Strathclyde, Glasgow, UK \\ euan.a.morris@strath.ac.uk
}

\begin{abstract}
Partial discharge monitoring is frequently used in AC cable systems, and there exists a strong desire for the same for DC cables within the electrical power industry, given their recent increased use. However, DC PD is a less well understood phenomenon. This paper provides analysis of three methods of partial discharge characterisation: pulse duration and amplitude analysis, frequency-domain spectra analysis, and partial discharge inception voltage analysis in artificially-created voids in polymeric cable insulation samples (polyethylene and polypropylene) under both $\mathrm{AC}$ and positive and negative DC excitations. From these a 'finger-print' of the defects can be determined based on the distribution of energy within the discharge frequency spectrum, and the inception voltage.
\end{abstract}

Index Terms-Partial Discharges, HVDC Insulation, Power Cables, Polypropylene Insulation, Polyethylene Insulation

\section{INTRODUCTION}

$\mathrm{P}$ ARTIAL DISCHARGE (PD) is increasingly a key component of the condition-based maintenance of electrical power cables used in AC transmission systems. Partial discharge under AC conditions has been a subject of study for several decades [1] with many commercial solutions available today [2]. Given the increasing use of HVDC links for subsea, and long-distance land-based transmission lines, there is a strong desire in the sector to utilise PD detection for these DC cables as well. However, PD under DC conditions and in DC cables is not a well understood phenomenon. Therefore, there are significant knowledge gaps which prevent the practical use of PD monitoring for HVDC cables.

This paper seeks to address this issue, by helping better identify the nature of DC PD. To achieve this, this paper discusses a method for the creation and PD testing of artificial voids in cable insulation samples and compares three methods of categorising the DC PD obtained by testing of three different void defect types- single void, two parallel voids, and two serial voids. The PD is categorised by comparing, first the duration and amplitudes of PD pulses, next the energy distribution through the frequency domain, and lastly from the partial discharge inception voltage (PDIV).

AC partial discharge and testing methods are defined in IEC standard 60270 "Partial Discharge Measurements", which describes partial discharge as "[a] localised electrical discharge that only partially bridges the insulation between conductors" [3].

$\mathrm{PD}$ monitoring under $\mathrm{AC}$ conditions can be used to determine the location and type of a defect in cable or other plant, by use of PDIV and partial discharge patterns, often analysed using phase-resolved partial discharge (PRPD) plots. However, this type of analysis is not possible under DC conditions, as there is no phase or equivalent to conduct such analysis therefore PD patterns are much less clear.

Initial research into PD under DC conditions was performed at the Technical University of Delft, Netherlands [4]. From this several differences between PD under AC were determined, including that DC discharges occur less frequently, are of smaller magnitude and, generally, are a symptom of a defect rather than a cause or contributing factor. Despite this, the analysis of DC PD is thought to be a useful indicator over the overall health of a cable and may be a possible indictor of other cables faults that might lead to cable failures.

In the past DC PD has been analysed by comparing the discharge amplitudes, and the time between pulses, by these, and other methods, research has determined common patterns for some defect types, including corona, surface and void discharges [5]-[7].

Within cables, partial discharges occur in defects in cable insulation that can be introduced during manufacture, installation or maintenance, void defects are among the most common.

For HVDC cables, the insulation itself is usually either polyethylene, historically low-density polyethylene (LDPE) and more recently cross-linked polyethylene (XLPE) [8], or polypropylene, in the form of a paper laminate (PPL) [9].

\section{METHOD}

The method used for the creation and testing of the insulation samples has been used for AC testing on polyethylene terephthalate (PET) [10], and for AC and DC testing on LDPE to determine PDIV and PD amplitudes and time between PD pulses [11], [12]. 


\section{A. Materials}

Materials were chosen to emulate the two most common types of modern HVDC cable, as discussed above.

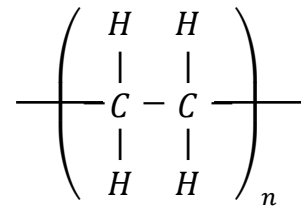

Fig. 1. Skeletal formula for polyethylene monomer.

Two polymers were tested. The first was LDPE (Fig. 1), which has a relative permittivity, $\varepsilon_{0}$, of 2.275 and a dielectric strength, $\mathrm{E}_{\max }$, of $27 \mathrm{kV} / \mathrm{mm}$, and the second was PP (Fig. 2) with a relative permittivity, $\varepsilon_{0}$, of 2.4 and a dielectric strength, $E_{\max }$, of $35 \mathrm{kV} / \mathrm{mm}$.

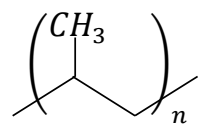

Fig. 2. Skeletal formula for polypropylene monomer.

\section{B. Test Samples}

Test samples of both LDPE and PP were created via a layering method. Layers measured $15 \mathrm{~mm} \times 15 \mathrm{~mm}$ and were $0.05 \mathrm{~mm}$ thick. Seven layers were used for each sample, giving a total thickness of $0.35 \mathrm{~mm}$. The artificial void was introduced by removing a circular section of diameter $1 \mathrm{~mm}$ from the centre layer.

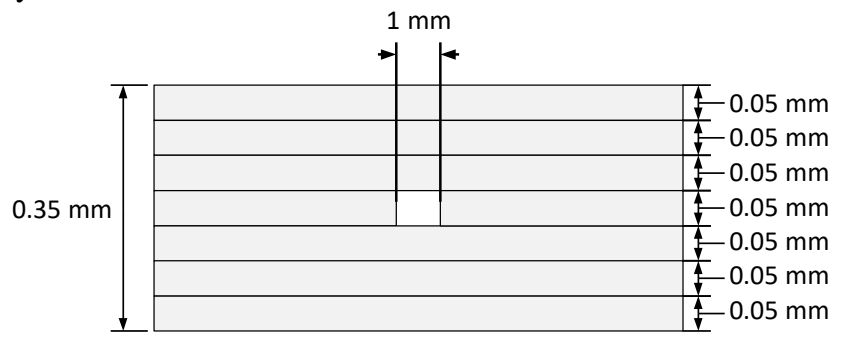

Fig. 3. Representation of layered artificial void sample. There are seven layers made from $0.05 \mathrm{~mm}$ thin film LDPE. (Not to scale.)

Layers were created with a single void, as in Fig. 3, with two voids a serial configuration (with one $1 \mathrm{~mm}$ void created in two layers with a single intact layer between then), and with two voids in a parallel configuration (with two $1 \mathrm{~mm}$ voids created on the same layer and space $1 \mathrm{~mm}$ apart).

\section{Test Rig}

The experimental test rig was comprised of two $7.5 \mathrm{~mm}$ diameter cylindrical brass electrodes. The bottom electrode was fixed, and connected to earth, and the height of the top electrode, which was connected to the HV supply, could be adjusted. The sample was placed in the test rig, with the void in the centre of the electrodes, and the electrode gap was adjusted to $0.35 \mathrm{~mm}$, the same thickness as the sample.

The remainder of the rig was constructed of ABS.

\section{Test Circuit}

For the AC testing two measurement systems were used. A LeCroy Waverrunner 104Xi was used for measurements in the time domain, and a Doble PDS200 was used for measurements in the frequency domain. Fig. 4 shows a circuit diagram of the system.

\section{0 kV Transformer}

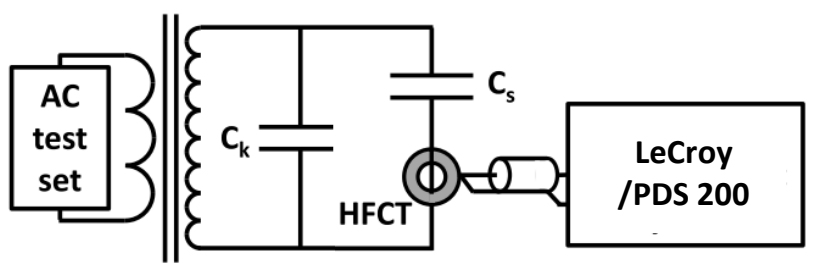

Fig. 4. AC test circuit including IEC 60270 PD detection system. The circuit includes the AC generator; transformer; $\mathrm{C}_{\mathrm{k}}$, a coupling capacitor; $\mathrm{Ca}$, the sample under test; and a HFCT connected to the detection systems

The same detection systems were used for the DC testing, with a basic high voltage rectifier used to produce a DC voltage. This configuration is shown in Fig. 5.

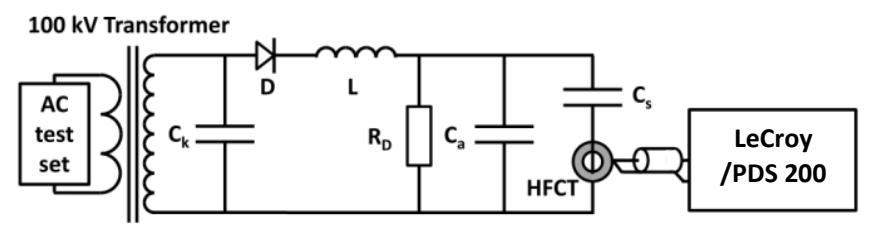

Fig. 5. DC test circuit including IEC 60270 PD detection system. The circuit includes the AC generator; transformer; $\mathrm{C}_{\mathrm{k}}$ and $\mathrm{C}_{\mathrm{S}}$, two coupling capacitors; $\mathrm{Ca}$, the sample under test; $\mathrm{D}$, an $\mathrm{HV}$ diode; $\mathrm{L}$, an $\mathrm{HV}$ inductor; $\mathrm{R}_{\mathrm{D}}$, a resistive divider for voltage measurement; and an HFCT connected to the detection systems

\section{E. Procedure}

The first stage in the testing procedure was to determine the PDIV. This was done by energising the system at its minimum voltage $(1.1 \mathrm{kV})$, then increasing the input by $100 \mathrm{~V}$ every ten minutes until the PDIV threshold (defined as 1 PD event occurring per minute) was reached. This procedure was the same for AC and DC voltages.

For PD testing, the sample was stressed at a voltage $10 \%$ greater than the PDIV. Measurements were taken after a 'settling down' period of 20 minutes. Testing was performed under AC, positive DC, and negative DC stresses.

\section{RESULTS AND DISCUSSION}

As discussed above, measurements were taken in both the time and frequency domains. In the time domain pulses were recorded, with the aim to extract the duration of these pulses and their amplitudes. In the frequency domain the spectrum of the discharges was recorded. To compare these spectra the energy components of the beginning of the recorded spectrum (50 MHz to $200 \mathrm{MHz}$ ) were compared to the middle (200-350 $\mathrm{Mhz}$ ) and the end (350 MHz to $500 \mathrm{MHz}$ ).

From both the time and frequency domain measurements, the aim was to identify a 'finger print' for each of the polymer type/voltage type/defect type combinations similar to [13] and [14].

However, the DC testing under both the positive and negative stresses was unable to induce PD in the polypropylene samples, therefore results are only presented for polypropylene under $\mathrm{AC}$ conditions, with AC, +DC and -DC presented for the lowdensity polyethylene. The partial discharge inception voltages (PDIV) were also recorded. 

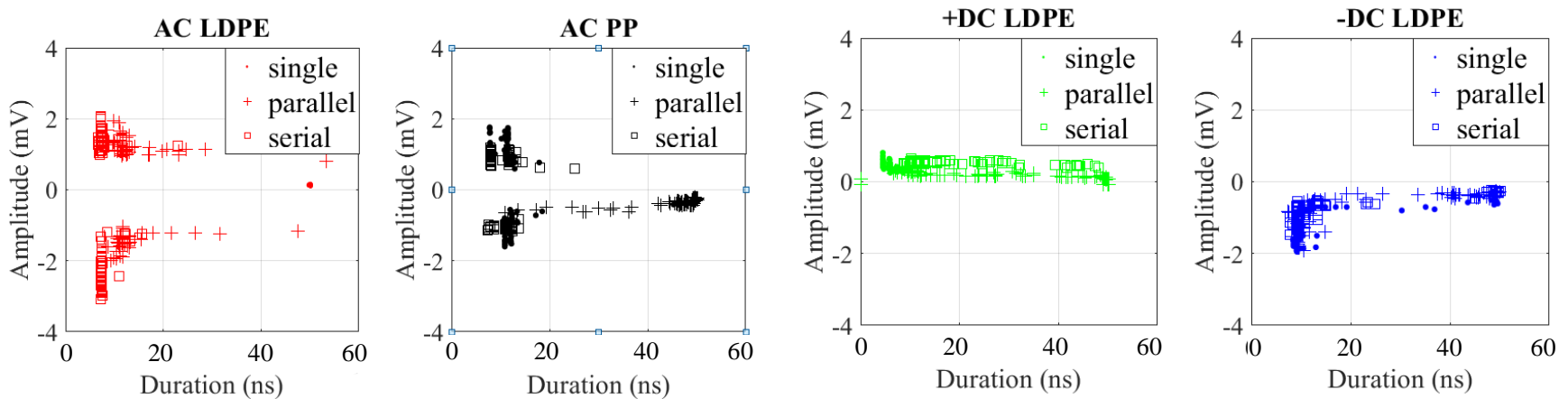

Fig. 6. Amplitude and Duration of PD Pulses for (1) AC voltage applied to LDPE samples, (2) AC voltage applied to PP samples, (3) positive DC voltage applied to LDPE samples, and negative DC voltage applied to LDPE samples. Single voids samples are indicated by $(\cdot)$, parallel voids by $(+)$ and serial by $(\square)$.

\section{A. Pulse Characteristics}

Comparing the plots shown Fig. 6 it is clear that the voltage type has little effect on pulse duration, as all plots show a range of pulse durations from 0 to 60 nanoseconds.

There is a clearer distinction between the defect types however, with the parallel voids under AC conditions in both the LDPE and PP samples, generally having a broader range of pulse durations, albeit in the LDPE with the majority of pulses being in the 0-20 ns range. Under AC conditions the single void and serial voids have similar characteristics, with almost all pulses falling into the same 0-20 ns range. The parallel void defect produces a more noticeable cluster in the PP, with most of pulses having a duration of 40-60 ns. Looking at the DC results there is a far less of a distinction between defect types under either the positive or negative stress, with all three producing a fairly even distribution of pulse durations across the 0-60 ns range. This suggests the use of pulse duration to attempt to identify defect types may not be of much practical use under DC conditions.

Considering pulse amplitudes, initially we see the expected characteristics, AC pulses occur both with positive and negative amplitudes, and DC pulses are unipolar in the opposite polarity to the applied stress. Under AC conditions the serial void configuration tends to produce pulses for a greater amplitude in the LDPE samples, while in the PP samples the single void configuration is dominant in this regard. Under positive DC conditions pulse amplitudes were generally in the order of $<1$ $\mathrm{mV}$, while negative DC conditions produced slightly higher magnitudes of $>-2 \mathrm{mV}$ for all three defect types. Overall it does not seem as through looking at the pulse characteristics are a suitable method for categorising defect type.

\section{B. Frequency characteristics}

To determine the characteristics of the partial discharge events in the frequency domain, the energy rates for the recorded PD for the beginning (50-200 MHz), middle (200-350 MHz) and end $(350-500 \mathrm{MHz})$ of the recorded frequency spectrums showing the proportion of the total energy that falls within each of these bands. From this Fig. 7 can be produced, which shows a ternary plot displaying this information.

From Fig. 7 several distinctions can be made between defect types under different voltage conditions.

There is a clear cluster of energy distributions for the parallel void configuration, with these spectra tending to have between $60-70 \%$ of their energy found in the first band. Between 30-
$40 \%$ is found in the second band, and the remaining $0-20 \%$ found in the end band for all voltage types. The serial void configurations are also distinct, both from the other defect types, but also from each other. The serial void in the PP has almost all of its energy found in the first band. In the LDPE sample the spectrum is also dominated by the first band, with $\sim 80 \%$ of the energy located here, and $\sim 20 \%$ in the final band, with very little in the middle band.

Both the positive and negative DC stresses applied to the LDPE produce spectra with around 25\% of their energy in the middle band, with the positive DC being more dominated by the middle band $(\sim 60 \%)$ and the negative DC being evenly split between the middle and end bands. The single void defect also produced very separate spectra. The AC stress (in both polymer types) is very dominated by the first band, while the negative DC is concentrated in the middle band, and the positive DC in the end band.

Overall this suggests the frequency domain analysis is a useful tool to distinguish defect types, provided the polymer type and voltage type are known (which is likely).

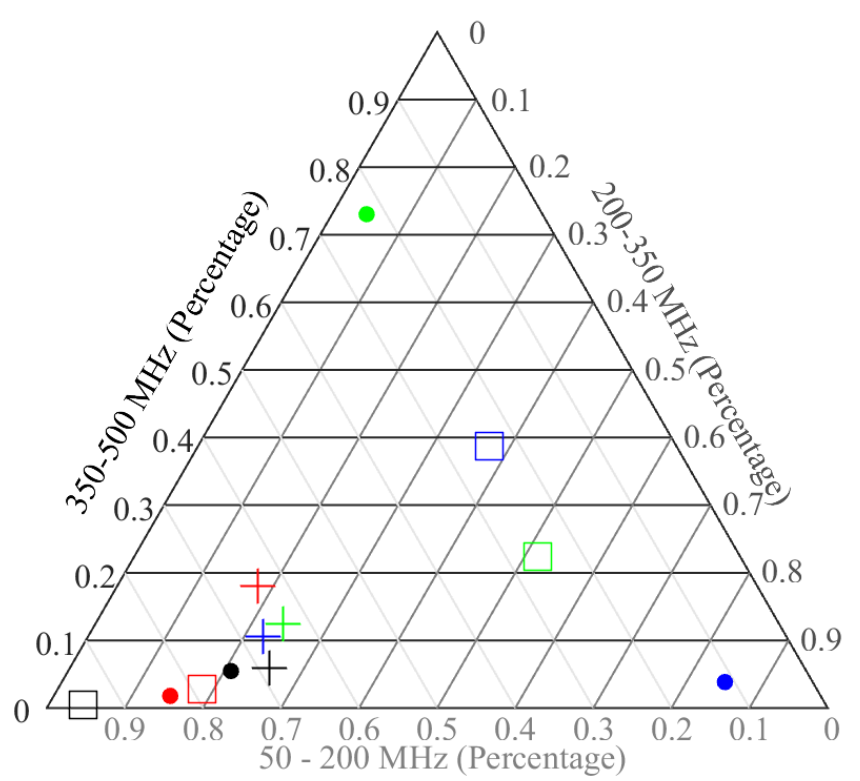

Fig. 7. Ternary plot showing energy rate of AC voltage applied to LDPE samples (red) AC voltage applied to PP (black), positive DC voltage applied to LDPE samples (green), and negative DC voltage applied to LDPE samples (blue).

Single voids samples are indicated by $(\cdot)$, parallel voids by $(+)$ and serial by $(\square)$. 


\section{PD Inception.}

The final PD characteristics that was investigated for use as an identifier, was the PD inception voltage (PDIV), which was defined as the voltage at which there was one recorded PD event per minute. Table 1 shows the PDIV values for each of the combinations of polymer, voltage and defect types. (AC voltages are rms values, while DC voltages are peak values).

TABLE I

PD INCEPTION VOLTAGES

\begin{tabular}{|c|c|c|c|c|}
\hline \multicolumn{2}{|c|}{} & \multicolumn{3}{|c|}{ Voltage Type } \\
\hline $\begin{array}{c}\text { Polymer } \\
\text { Type }\end{array}$ & $\begin{array}{c}\text { Defect } \\
\text { Type }\end{array}$ & AC & + +DC & -DC \\
\hline \multirow{4}{*}{ LDPE } & $\begin{array}{c}\text { Single } \\
\text { Void }\end{array}$ & $4.1 \mathrm{kV}$ & $18.6 \mathrm{kV}$ & $18.6 \mathrm{kV}$ \\
\cline { 2 - 5 } & $\begin{array}{c}\text { Parallel } \\
\text { Voids }\end{array}$ & $3.5 \mathrm{kV}$ & $15.0 \mathrm{kV}$ & $14.1 \mathrm{kV}$ \\
\cline { 2 - 5 } & $\begin{array}{c}\text { Serial } \\
\text { Voids }\end{array}$ & $2.9 \mathrm{kV}$ & $17.7 \mathrm{kV}$ & $21.1 \mathrm{kV}$ \\
\hline \multirow{4}{*}{ PP } & $\begin{array}{c}\text { Single } \\
\text { Void }\end{array}$ & $3.9 \mathrm{kV}$ & N/A & N/A \\
\cline { 2 - 5 } & $\begin{array}{c}\text { Parallel } \\
\text { Voids }\end{array}$ & $3.3 \mathrm{kV}$ & N/A & N/A \\
\cline { 2 - 5 } & $\begin{array}{c}\text { Serial } \\
\text { Voids }\end{array}$ & $2.8 \mathrm{kV}$ & N/A & N/A \\
\hline
\end{tabular}

As expected, the PDIV under DC conditions was greater than that under $\mathrm{AC}$ conditions, due to the differences in the mechanisms of discharges. The PDIV was also lower when multiple defects were present, excluding the serial voids under negative DC conditions. The PDIV was generally lower in the PP under AC conditions, however under DC conditions was higher than the breakdown strength of the material, possibly due to the availability of electrons in the PP as compared to the LDPE, although further research is suggested to confirm this. Overall, the PDIV may have utility in determining defect type, especially when coupled with the above frequency analysis.

\section{CONCLUSION}

Layered samples of both LDPE and PP were created to simulate voids in HV cable insulation. PDIV was determined, and PD data was gathered by subjecting the samples to electrical stress. Three methods of creating a 'finger print' to identify defect types (single void, serial voids and parallel voids), were investigated. The first method, comparing the pulse amplitudes and durations was not found to be useful in identifying defect types. The second, looking at the energy rates of the frequency domain spectra of the PD, across the beginning, middle and end frequencies, was more successful, with clear differences between the defects at both AC and DC stresses found. The final method of comparing the PDIV, was found to have some utility, albeit less than the frequency method.

From this it is found that analysis in the frequency domain is the most effective method of PD analysis under HVDC conditions, and also has utility under AC conditions. Further work could be undertaken to compare this method of PD characterization to conventional AC PD analysis methods such as phase-resolved partial discharge plots.
Additional planned future work on DC PD, includes the investigation of faults in cable samples, to determine if this method has practical applications in cable monitoring systems, as well as looking at a broader range of faults. Another area of interest would be how the DC PD 'finger print' develops over time as the cable defect is subjected to continued stress, with the ultimate goal of determining when a PD source is likely to cause a cable failure.

\section{ACKNOWLEDGEMENT}

The authors would like to thank: from the University of Strathclyde- Edward Corr, Rong Tang, and Brian Stewart; from Glasgow Caledonian University- Donald Hepburn; and from Scottish \& Southern Electricity Networks- Geary Cleary, and David Stroud, for their advice on the direction and implementation of the project.

All data created during research published in this paper are openly available at: http://dx.doi.org/10.15129/648537692b98-49fb-afd9-0a857a0ca392.

\section{REFERENCES}

[1] R. Bartnikas, "Partial Discharges: Their Mechanism, Detection and Measurement," IEEE Trans. Dielectr. Electr. Insul., vol. 9, no. 5, pp. 763-808, 1997.

[2] HVPD, "HVPD Products and Services Catalogue." [Online]. Available: www.hvpd.co.uk. [Accessed: 25-May-2017].

[3] International Electrotechnical Commission - Technical Commitee 42 -High-voltage and high-current test techniques, IEC 60270:2000 High-voltage test techniques - Partial discharge measurements, no. 3. 2000, pp. 1-99.

[4] P. H. F. Morshuis and J. J. Smit, "Partial discharges at de voltage: Their mechanism, detection and analysis," IEEE Trans. Dielectr. Electr. Insul., vol. 12, no. 2, pp. 328-340, Apr. 2005.

[5] T. Klueter, J. Wulff, and F. Jenau, "Measurement and Statistical Analysis of Partial Discharges at DC Voltage," in Universities Power Engineering Conference, 2013, vol. 0, pp. 0-4.

[6] E. Corr, W. H. Siew, and W. Zhao, "Long Term Testing and Analysis of Dielectric Samples Under DC Excitation," in IEEE Electrical Insulation Conference, 2016, pp. 2-5.

[7] K. Suenage, K. Uchida, and N. Hozumi, "Location of Water Tree Degraded Point Along XLPE Cable Line Using DC Voltage," 2008 Int. Conf. Cond. Monit. Diagnosis, Beijing, China, April 21-24, 2008, pp. 2-5, 2008.

[8] G. Mazzanti, "Problems in HVDC Extruded Cable systems," in HubNet Colloquium on Power Cables and Cable Integrity, 2016.

[9] R. Hata, "Solid DC submarine cable insulated with polypropylene laminated paper (PPLP)," SEI Tech. Rev., no. 62, pp. 3-9, 2006.

[10] D. Adhikari, D. M. Hepburn, and B. G. Stewart, "PD characteristics and degradation in PET insulation with vented and unvented internal voids," Electr. Power Syst. Res., vol. 100, pp. 65-72, 2013.

[11] E. A. Morris and W. H. Siew, "Partial Discharge Activity in Polymeric Cable Insulation under High Voltage AC and DC," in 52nd International Universities' Power Engineering Conference, 2017, pp. $1-4$.

[12] E. A. Morris and W. H. Siew, "A Comparison of AC And DC Partial Discharge Activity in Polymeric Cable Insulation," in 21st IEEE International Pulsed Power Conference, 2017, pp. 1-4.

[13] P. C. Baker, M. D. Judd, and S. D. J. McArthur, "A frequency-based RF partial discharge detector for low-power wireless sensing," IEEE Trans. Dielectr. Electr. Insul., vol. 17, no. 1, pp. 133-140, 2010.

[14] W. Gao, D. Ding, W. Liu, and X. Huang, "Analysis of the intrinsic characteristics of the partial discharge induced by typical defects in GIS," IEEE Trans. Dielectr. Electr. Insul., vol. 20, no. 3, pp. 782790, 2013. 